\title{
Rumiación, crecimiento y sintomatología postraumática en personas que han vivido experiencias altamente estresantes
}

\author{
Rumination, Posttraumatic Growth and Posttraumatic Symptoms \\ in People Who Have Lived Highly Stressful Experiences \\ Ruminação, crescimento e sintomatologia pós-traumática em pessoas \\ que tem vivido experiências altamente estressantes
}

\author{
Felipe E. García* \\ Nicole Vega Rojas* \\ Francisco Briones Araya* \\ Yasmín Bulnes Gallegos* \\ Universidad Santo Tomás, Concepción, Chile.
}

Doi: http://dx.doi.org/10.12804/revistas.urosario.edu.co/apl/a.4983

\section{Resumen}

Los eventos altamente estresantes producen consecuencias en la salud mental, principalmente negativas, pero en el último tiempo se han descrito algunas positivas, como la posibilidad de aprender a partir de la experiencia. De ese modo, se hace necesario establecer qué variables son capaces de predecir una u otra consecuencia. El presente estudio analizó la influencia de distintos tipos de rumiación (negativa, reflexiva, intrusiva y deliberada), y la severidad subjetiva del evento sobre el crecimiento postraumático $-\mathrm{CPT}-\mathrm{y}$ la sintomatología postraumática — STP- en personas que experimentaron un evento altamente estresante.
Participaron 629 personas mayores de 18 años (51\% hombres), habitantes de la provincia de Concepción, Chile. A través de un análisis de sendero se evaluó un modelo hipotético que mostraba que las rumiaciones reflexiva y deliberada influían sobre el CPT, que las rumiaciones negativa e intrusiva influían sobre la SPT, que la rumiación deliberada mediaba entre las otras formas de rumiación y la severidad subjetiva con el CPT, y que la rumiación intrusiva mediaba la relación entre la severidad subjetiva y la rumiación negativa con la SPT. Los índices de ajuste del modelo fueron satisfactorios. Un análisis multigrupo mostró que no había diferencias en el modelo entre hombres y mujeres. Se discute la relevancia de estos hallazgos para la predicción de

* $\quad$ Felipe E. García, Nicole Vega Rojas, Francisco Briones Araya, Yasmín Bulnes Gallegos, Facultad de Ciencias Sociales y Comunicaciones, Universidad Santo Tomás, Concepción, Chile.

La correspondencia relativa a este artículo debe ser enviada a Felipe E. García, Escuela de Psicología, Universidad Santo Tomás, Av. Prat 855, Concepción, Chile. Correo electrónico: fgarcia@santotomas.cl

Cómo citar este artículo: García, F. E., Vega Rojas, N., Briones Araya, F. \& Bulnes Gallegos, Y. (2018). Rumiación, crecimiento y sintomatología postraumática en personas que han vivido experiencias altamente estresantes. Avances en Psicología Latinoamericana, 36(3), 443-457. DOI: http://dx.doi.org/10.12804/revistas.urosario.edu.co/apl/a.4983 
consecuencias tras un evento altamente estresante, y su relación con la prevención e intervención psicológica en personas expuestas.

Palabras clave: pensamiento repetitivo, trastorno de estrés postraumático, estrategias de afrontamiento, resiliencia, análisis multigrupo.

\section{fibstract}

Highly stressful events produce consequences on mental health; while mainly negative, recent findings suggest some positive consequences such as learning from one's own experience. This study analyzed the influence of different types of rumination (brooding, reflection, intrusive and deliberate) and the subjective severity of event on posttraumatic growth - PTGand posttraumatic symptoms - PTS - in people who experienced a highly stressful event. Six hundred and twenty-nine people participated in the study, all older than 18 years ( $51 \%$ men), inhabitants of the province of Concepcion, Chile. A hypothetical model was evaluated through a path analysis which showed that reflective and deliberate rumination influenced on the PTG, the negative and intrusive rumination influenced on the PTS, that deliberate rumination mediated the relationship between other forms of rumination and subjective severity with the PTG, and intrusive rumination mediated the relationship between the subjective severity and the negative rumination with the PTS. The model fit indices were satisfactory. A multi-group analysis showed no difference in the model between men and women. Finally, we discuss the relevance of these findings for the prediction of consequences after a highly stressful event and its relation to the prevention and psychological intervention in persons exposed.

Keywords: Repetitive thinking, post-traumatic stress disorder, coping, resilience, multi-group analysis.

\section{Resumo}

O presente estudo analisou a influência de distintos tipos de ruminação (negativa, reflexiva, intrusiva e deliberada), e a severidade subjetiva do evento sobre o crescimento pós-traumático - $\mathrm{CPT}-\mathrm{e}$ a sintomatologia pós-traumática — STP — em pessoas que experimentaram um evento altamente estressante. Participaram 629 pessoas maiores de 18 anos (51\% homens), habitantes da província de Concepción, Chile. Através de uma análise de trilha se avaliou um modelo hipotético que mostrava que as ruminações reflexiva e deliberada influíam sobre o CPT, que as ruminações negativa e intrusiva influíam sobre a SPT, que a ruminação deliberada mediava entre as outras formas de ruminação e a severidade subjetiva com o CPT, e que a ruminação intrusiva mediava a relação entre a severidade subjetiva e a ruminação negativa com a SPT. Os índices de ajuste do modelo foram satisfatórios. Uma análise multigrupo mostrou que não havia diferenças no modelo entre homens e mulheres. Discute-se a relevância destes resultados para a predição de consequências após um evento altamente estressante, e sua relação com a prevenção e intervenção psicológica em pessoas expostas.

Palavras-chave: pensamento repetitivo, transtorno de estresse pós-traumático, estratégias de afrontamento, resiliência, análise multigrupo.

Los eventos altamente estresantes son alteraciones en la historia de vida de un individuo que generan la necesidad de adaptarse (Sutin, Costa, Wethington \& Eaton, 2010). Estos eventos son potencialmente traumáticos cuando su exposición implica una amenaza para su integridad física o la de los demás (American Psychiatric Association, 2000). Una de las consecuencias posibles de estos eventos es el desarrollo de estrés postraumático, ya sea en forma de trastorno - TEPT - o de sintomatología - SPT- Sin embargo, esta no es la única consecuencia posible, las personas también pueden responder frente al mismo evento como si fuera un desafío a superar, generando aprendizaje a partir de la lucha que emprenden para enfrentar los efectos que dichos eventos han tenido en sus vidas, lo que se denomina crecimiento postraumático — CPT— (Tedeschi \& Calhoun, 1996).

En los procesos psicológicos que se desencadenan tras un evento altamente estresante resulta 
relevante la percepción subjetiva de la amenaza, también llamada severidad subjetiva del evento (García, Reyes \& Cova, 2014). La evidencia disponible nos muestra que la severidad subjetiva resulta un predictor más fuerte ya sea de la SPT o del CPT que los aspectos más objetivos del evento (Boals \& Schuettler, 2009; Morris, Shakespeare-Finch, Rieck \& Newberry, 2005).

Debido a que tanto la SPT como el CPT se desencadenan tras la percepción de un evento como severo, los estudios muestran una fuerte correlación positiva entre las tres variables (Boals \& Schuettler, 2009; García, Jaramillo, Martínez, Valenzuela \& Cova, 2014; Hafstad, Gil-Rivas, Kilmer \& Raeder, 2010; Kilmer \& Gil-Rivas, 2010; McCaslin et al., 2009; Morris et al., 2005). Esta correlación ha llevado a un cuestionamiento respecto a la supuesta naturaleza adaptativa del CPT (véase Vázquez, Castilla \& Hervás, 2009). De ese modo, surge la necesidad de estudiar los caminos que conducen al SPT o al CPT y discriminar qué estrategias llevan a uno u otro resultado.

De las estrategias estudiadas en su relación con las consecuencias posestrés o postrauma destaca la rumiación, un proceso cognitivo consistente en pensar repetidamente sobre el evento vivido o sus consecuencias (Calhoun, Cann \& Tedeschi, 2010). La rumiación es una clase de pensamiento consciente que gira en torno a un tema fundamental común y se repite en ausencia de demandas ambientales inmediatas (Papageorgiou \& Wells, 2004). Al respecto existen dos tipos de rumiación que han sido estudiados por la literatura científica en su relación con la salud mental. El primero apunta al contenido de estos pensamientos y se clasifica en rumiación negativa (o brooding) y rumiación reflexiva (Treynor, Gonzalez \& Nolen-Hoeksema, 2003). El segundo apunta al control ejercido sobre estos pensamientos y se clasifica en rumiación intrusiva y deliberada (Cann et al., 2011).

Las rumiaciones negativa y reflexiva tienen un carácter más estable en la persona en forma de estilos de pensamiento, que se han asociado tradicionalmente a la presencia de sintomatología depresiva (Nolen-Hoeksema, 1987). En la rumiación reflexiva la persona piensa por qué se siente así como acto de introspección sobre el ánimo depresivo para obtener una solución del problema, analizar las dificultades vividas e intentar entender qué es lo que lo lleva a sentirse de una determinada forma. En la rumiación negativa se revive la sintomatología depresiva en forma pasiva, focalizándose en el malestar experimentado, lo que se puede asociar a una evaluación negativa de sí mismo (Cova, Rincón \& Melipillán, 2009). Kane (2009) señala que la rumiación negativa es un tipo de afrontamiento en forma de evitación relacionada a otras consecuencias negativas aparte de la depresión, como la SPT.

Por otro lado, las rumiaciones intrusiva y deliberada suelen estar asociadas a eventos altamente estresantes o potencialmente traumáticos (Cann et al., 2011). La intrusiva ha sido definida como irrupciones involuntarias de pensamientos sobre la experiencia que uno no escoge traer a la mente. La deliberada se refiere a pensamientos intencionados sobre un evento, que se realizan de forma voluntaria y pueden enfocarse decididamente en tratar de entender los eventos y sus implicancias (Cann et al., 2011). Se ha observado que la rumiación tanto deliberada como intrusiva se activan cuando la persona percibe un evento como estresante y por ello en algunos estudios ambas se relacionan positivamente tanto con la SPT como con el CPT, aunque con distinta fuerza (Alzugaray, García, Reyes \& Álvarez, 2015; Danhauer et al., 2013).

Por otro lado, los estudios que buscan determinar la influencia diferencial de los procesos de rumiación en la SPT y CPT nos muestran que efectivamente estos son distintos: las rumiaciones negativa e intrusiva serían un factor de vulnerabilidad en el desarrollo y mantenimiento de la SPT y las rumiaciones deliberada y reflexiva tendrían una influencia más fuerte en el CPT (Alzugaray et al., 2015; García, Cova, Rincón \& Vázquez, 2015; Soo \& Sherman, 2015; Triplett, Tedeschi, Cann, 
Calhoun \& Reeve, 2012). En el estudio longitudinal de García, Duque y Cova (2017), la rumiación intrusiva predice SPT y la deliberada predice CPT, controlando los otros tipos de rumiación. La negativa y la intrusiva actuarían incluso inhibiendo el desarrollo de CPT (Stockton, Hunt \& Joseph, 2011; Taku, Cann, Tedeschi \& Calhoun, 2009).

El modelo teórico de Calhoun et al. (2010) propone que, para lograr un crecimiento tras un evento traumático, los procesos más involuntarios de pensamiento repetitivo deberían dar el paso a procesos más voluntarios. En otras palabras, la rumiación deliberada debería actuar como mediadora entre la rumiación intrusiva y el CPT. Empíricamente, se ha observado que a medida que la rumiación intrusiva da paso a una más deliberada, se llega al СРт (García et al., 2015; García, Cova, Rincón, Vázquez \& Páez, 2016; García, Jaramillo et al., 2014; Triplett et al., 2012).

La fuerte relación entre rumiación deliberada y el CPT se ha encontrado en muchos estudios (Avilés, Cova, Bustos \& García, 2014; Allbaugh, 2013; Lindstrom, Cann, Calhoun \& Tedeschi, 2013), ya que la primera es una de las variables que con mayor probabilidad conducen al segundo. Esto se debería a que la rumiación voluntaria ayudaría a la reevaluación para encontrar aspectos positivos y beneficios de la experiencia vivida $(\mathrm{Su}$ \& Chen, 2015), mediando incluso su relación con otras variables, como la severidad subjetiva y la rumiación negativa (García et al., 2015; García et al., 2016). Por otro lado, la rumiación intrusiva también mediaría la relación entre otras variables, como el desafío que el evento representa para las creencias centrales (Triplett et al., 2012) o la severidad del trauma (Morris \& Shakespeare-Finch, 2011), con la SPT.

El presente estudio pretende analizar y conocer el efecto que producen los distintos tipos de rumiación en el desarrollo del CPT o de la SPT, a través de un análisis de los caminos diferenciales que llevan a una u otra consecuencia, pretendiendo mostrar que las rumiaciones negativa e intrusiva conducen a la SPT, que la rumiaciones reflexiva y deliberada conducen al CPT, que la rumiación intrusiva media la relación de la severidad subjetiva y la rumiación negativa con la SPT, y que la rumiación deliberada media la relación de la severidad subjetiva, la rumiación reflexiva y la rumiación intrusiva con el CPT. El modelo hipotetizado se observa en la figura 1.

\section{Método}

\section{Diseño}

Se utilizó un diseño cuantitativo de tipo descriptivo y correlacional. Las mediciones se llevaron a cabo en un solo momento, por lo que se trata de un estudio trasversal.

\section{Participantes}

Participaron 629 personas adultas, 505 personas de población general que señalaron haber experimentado un evento altamente estresante en los últimos cuatro años y 124 trabajadores que sufrieron un accidente laboral reciente, todos habitantes de la provincia de Concepción, Chile. De ellos, 308 fueron mujeres (49\%) y 321 hombres (51\%). La media total de edad fue de 38.47 años $(D E=13.85)$ con un rango desde los 18 a los 83 años. El evento estresor ocurrió desde 1 a 48 meses al momento de la evaluación $(M=14.79$ y $D E=$ 11.84). En cuanto al tipo de estresor, un $26.2 \%$ fue un accidente laboral o de tránsito, un $17.2 \%$ sufrió la muerte de una persona cercana, un $10.8 \%$ fue afectada por la enfermedad grave de una persona cercana, un $9.7 \%$ vivió la ruptura de una pareja, y un $36.1 \%$ se expuso a otros eventos estresantes de menor frecuencia.

\section{Instrumentos}

Escala de severidad subjetiva del evento — SSE — : es una escala para detectar la severidad 
con la que se percibe el evento estresante (Alzugaray et al., 2015). Consta de tres ítems que evalúan si la persona percibe que el evento alteró su vida, si lo siente como traumático y si piensa que es grave lo que le ha pasado. Se responde en una escala Likert en un rango que va de 0 a 4 puntos; en el presente estudio mostró una consistencia interna adecuada $(\alpha=0.86)$.

Escala de respuesta rumiativa - RRS-: fue propuesta por Treynor et al. (2003) para evaluar la rumiación reflexiva y negativa. Fue validada en Chile por Cova, Rincón \& Milipillán (2007), quienes conservaron los 10 ítems de la escala original, agregando dos más para cada escala, dejando el instrumento en 14 ítems. Se responde en una escala Likert que va desde 0 (casi nunca) a 3 (casi siempre). En el estudio de Cova et al. (2007) la escala mostró validez de constructo en la distinción entre las dos formas de rumiación. En el presente estudio se observan indicadores satisfactorios de confiabilidad, tanto en reflexión $(\alpha=0.79)$ como en rumiación negativa ( $\alpha=0.87$ ),

Escala de rumiación relacionada al evento ERRI - : esta escala fue creada por Cann et al. (2011) y mide las rumiaciones intrusiva y deliberada. Fue validada para su uso en población chilena por Alzugaray et al. (2015), quienes presentaron una versión más breve de 12 ítems, 7 para rumiación intrusiva y 5 para deliberada. Se responde en una escala Likert desde 0 (casi nunca) a 3 (casi siempre). La versión de Alzugaray et al. (2015) mostró validez de constructo al diferenciar entre ambos factores. En el presente estudio obtuvo una consistencia interna adecuada, para rumiación tanto intrusiva $(\alpha=0.92)$ como deliberada $(\alpha=0.80)$.

SPRINT-E: fue creada por Connor y Davidson (2001) para evaluar sintomatología postraumática, según los criterios del DSM-IV. Fue validada en Chile por Leiva-Bianchi y Gallardo (2013). Posee 12 preguntas que se responden en una escala Likert desde 0 (nada) a 3 (mucho). En el presente estudio se obtuvo una confiabilidad alta para esta escala $(\alpha=0.91)$.
Inventario de crecimiento postraumático, versión breve - PTGI-SF-: fue creado por Cann et al. (2010), a partir del inventario de crecimiento postraumático de Tedeschi y Calhoun (1996). Esta versión breve fue validada para su uso en población chilena por García y Wlodarczyk (2016). Está compuesto de 10 ítems que se responden en una escala Likert de seis alternativas, desde 0 (ningún cambio) hasta 5 (un cambio muy importante). En el estudio de García y Wlodarczyk (2016) se obtuvo un $\alpha=0.94$.

Cuestionario socio-demográfico: se incluyó un cuestionario con preguntas relativas al género y la edad, además de una pregunta dicotómica (Sí/No) para indicar si ha vivido en los últimos 4 años un evento altamente estresante y un listado para seleccionar el tipo de evento vivido entre 11 opciones, basadas en Norris, Hamblen, Brown y Schinka (2008): desastre natural, enfermedad grave personal, enfermedad grave de una persona muy cercana, accidente hogareño, accidente laboral o de tránsito, ruptura de pareja, violencia del Estado, violencia doméstica, violencia delictual, entre otros.

\section{Procedimiento}

Los participantes se seleccionaron a través de dos tipos de método. Un primer grupo fue escogido a través de un muestreo, en ocasiones sucesivo, con un diseño muestral de panel. Una muestra inicial de 1124 personas fue seleccionada aleatoriamente desde la población general; de ellos, 505 personas declararon haber vivido un evento altamente estresante en los últimos cuatro años, por lo que fueron incluidos en la muestra final. No hubo personas que se negaran a responder.

El segundo grupo de participantes fue seleccionado desde un listado de personas accidentadas recientemente, con una licencia médica superior a 10 días y cuya atención médica fue realizada en un hospital especializado en salud laboral de Concepción (población objetivo). Del listado total 
de 152 personas que cumplieron con los requisitos de inclusión a lo largo de tres meses, 28 de ellos fueron excluidos debido a que no fueron ubicados en los teléfonos suministrados por el hospital y dos de ellos se negaron a responder, por lo que el grupo descendió a 124 personas (población marco).

Los participantes fueron encuestados por estudiantes de psicología previamente capacitados. Los cuestionarios fueron autoadministrados en presencia del encuestador, previa firma de una carta de consentimiento informado en la cual se garantizaba la confidencialidad, resguardo de los datos entregados y participación voluntaria. Este estudio fue aprobado con la Comisión de Ética de la Universidad Santo Tomás, Chile. (n. $\left.{ }^{\circ} 89 / 2014\right)$ y por la Comisión de Ética de la Mutual de Seguridad, Chile (n. $\left.{ }^{\circ} 03 / 15\right)$.

\section{Análisis de datos}

Tras realizar pruebas de normalidad, se procedió a analizar los datos descriptivos de las variables independientes y dependientes, y a comparar por sexo a través de la $t$ de student para grupos independientes.

Se utilizó la correlación $r$ de Pearson y la regresión lineal múltiple — RLM- para estimar la influencia que tienen los distintos tipos de rumiación en el CPT y la SPT.

Finalmente, se utilizó un análisis de sendero para probar el modelo hipotetizado señalado en la figura 1. Para ello se usó el método de estimación de máxima verosimilitud, previo análisis del supuesto de normalidad multivariante a través del coeficiente de Mardia. Se utilizaron los siguientes índices y criterios de ajuste (Hu \& Bentler, 1999; Yu, 2002): 1) $\chi 2$ : valores no significativos indican un buen ajuste; 2) $\chi^{2 / g 1}$ : un buen ajuste está indicado por valores menores a 2; 3 ) Comparative Fit Index — CFI - y Tucker-Lewis Index - TLI-: un ajuste aceptable está dado por valores $\geq 0.90 \mathrm{y}$ un buen ajuste por valores $\geq 0.95$; 4) Root Mean Square Error of Approximation —RMSEA—: un ajuste aceptable está indicado por valores $\leq 0.08$ $(90 \% \mathrm{CI} \leq 0.10)$ y un buen ajuste por valores $\leq$ $0.05(90 \% \mathrm{CI} \leq 0.08)$.

El análisis de sendero muestra coeficientes path o efectos directos que son análogos a los puntajes beta de las RLM, y que indican en qué medida un cambio en la variable al comienzo de una flecha se relaciona con una alteración en la variable al final de la flecha (Aron y Aron, 2001). Los efectos indirectos son aquellos que relacionan dos variables a través de terceras. La mediación ocurre cuando el efecto indirecto entre dos variables es significativo (Pérez, Medrano \& Sánzhez-Rosas, 2013). La magnitud de los efectos indirectos se estima al multiplicar los coeficientes path existentes a lo largo de la línea causal entre dos variables (Arbuckle, 2003). Para todos estos análisis se utilizó el software estadístico SSPS Statistics 23 y el Amos v18 de IBM.

\section{Resultados}

Se observan unos coeficientes de asimetría entre los rangos de -0.09 a 0.77 y de curtosis entre -0.84 y 0.07 , puntajes aceptables para considerar que cada variable se distribuye normalmente; la prueba de Kolmogorov-Smirnov ratifica estos resultados.

En la tabla 1 se detallan los estadísticos descriptivos de las variables estudiadas en toda la muestra y diferenciadas por sexo. La comparación de mujeres y hombres encuentra diferencias significativas en severidad subjetiva, rumiación intrusiva у СРТ, obteniendo las mujeres puntajes más altos que los hombres.

Se realiza un análisis de correlación bivariada entre las variables, obteniendo los resultados que se detallan en la tabla 2. En esta se observa que la SPT se correlaciona significativamente con todas las variables, sin embargo, existe una correlación mayor con la rumiación intrusiva $(r=0.70)$ y la negativa $(r=0.62)$. Por otra parte, se observa que el CPT también correlaciona positivamente con 
Tabla 1

Estadisticos descriptivos para la muestra total $(n=629)$ y según sexo (mujeres $=308$; hombres $=321)$ y prueba t de student para la comparación según sexo

\begin{tabular}{|c|c|c|c|c|c|c|c|c|c|}
\hline & \multirow{2}{*}{ Mín. } & \multirow{2}{*}{ Máx. } & \multicolumn{2}{|c|}{ Total } & \multicolumn{2}{|c|}{ Mujeres } & \multicolumn{2}{|c|}{ Hombres } & \multirow{2}{*}{$t$} \\
\hline & & & $M$ & $D E$ & $M$ & $D E$ & $M$ & $D E$ & \\
\hline Severidad subjetiva & 0 & 12 & 6.65 & 3.17 & 7.12 & 3.07 & 6.20 & 3.19 & $3.71 * * *$ \\
\hline Rumiación negativa & 0 & 21 & 8.49 & 5.25 & 8.80 & 5.18 & 8.18 & 5.31 & 1.47 \\
\hline Rumiación reflexiva & 0 & 21 & 8.31 & 4.61 & 8.61 & 4.39 & 8.03 & 4.80 & 1.58 \\
\hline Rumiación deliberada & 0 & 15 & 6.44 & 3.92 & 6.90 & 3.55 & 6.51 & 3.74 & 1.31 \\
\hline Rumiación intrusiva & 0 & 21 & 7.73 & 5.56 & 8.31 & 5.52 & 7.16 & 5.55 & $2.61 * *$ \\
\hline Sintomatología postraumática & 0 & 36 & 10.6 & 7.89 & 10.79 & 8.22 & 10.44 & 7.63 & 0.42 \\
\hline Crecimiento postraumático & 0 & 50 & 27.09 & 13.29 & 29.35 & 13.43 & 24.92 & 12.80 & $4.24 * * *$ \\
\hline
\end{tabular}

$* * \mathrm{p}<0.01 ; * * \mathrm{p}<0.001$

Tabla 2

Correlación bivariada entre las variables de estudio $(N=629)$

\begin{tabular}{|c|c|c|c|c|c|c|c|}
\hline & 1 & 2 & 3 & 4 & 5 & 6 & 7 \\
\hline 1. Severidad subjetiva & - & $0.34 *$ & $0.30 *$ & $0.45^{*}$ & $0.46^{*}$ & $0.49 *$ & $0.22 *$ \\
\hline 2. Rumiación negativa & & - & $0.69 *$ & $0.55^{*}$ & $0.71 *$ & $0.62 *$ & $0.19 *$ \\
\hline 3. Rumiación reflexiva & & & - & $0.54 *$ & $0.55^{*}$ & $0.46^{*}$ & $0.26^{*}$ \\
\hline 4. Rumiación deliberada & & & & - & $0.64 *$ & $0.58^{*}$ & $0.41 *$ \\
\hline 5. Rumiación intrusiva & & & & & - & $0.70 *$ & $0.24 *$ \\
\hline 6. Sintomatología postraumática & & & & & & - & $0.21 *$ \\
\hline 7. Crecimiento postraumático & & & & & & & - \\
\hline
\end{tabular}

$* \mathrm{p}<0.001$

todas las variables, aunque en menor grado que con la SPT. La correlación más fuerte se presenta con la rumiación deliberada $(\mathrm{r}=0.41)$.

A continuación, se realizaron las RLM para conocer la influencia relativa de las variables independientes sobre la SPT y el CPT (tabla 3). Los resultados muestran que el modelo predictor de SPT es significativo $\left(F_{(5.620)}=78.9, p>0.001 ; \mathrm{R}^{2} \mathrm{adj}\right.$ $=0.53)$ y que las variables que influyen en forma directa son las rumiaciones intrusiva, negativa y deliberada, y la severidad subjetiva. El modelo predictor de CPT es también significativo $\left(F_{(5.620)}\right.$ $\left.=27.40, p>0.01 ; R^{2} a d j=0.17\right)$ y las variables que influyen en forma directa son las rumiaciones reflexiva y deliberada.

Con la observación de que las relaciones existentes entre las variables eran las esperadas, se procedió a realizar el análisis de sendero. Primero se observó la normalidad multivariante, obteniendo un coeficiente de Mardia de 5.46, procediendo a realizar el análisis a través del método de estimación de máxima verosimilitud. Se obtuvieron los siguientes índices de ajuste: 1) $\chi 2=192.06, p<$ 0.001 ; 2) $\mathrm{CFI}=0.99$; 3) $\mathrm{TLI}=0.98$; 4) $\mathrm{RMSEA}=0.06$ (IC $=0.05-0.06)$. Según los parámetros ya señalados, estos índices se consideraron satisfactorios, lo 
Tabla 3

Regresión lineal múltiple de las variables de estudio sobre la sintomatología y el crecimiento postraumático $(n=629)$

\begin{tabular}{lcccc}
\hline & \multicolumn{2}{c}{ Sintomatología postraumática } & \multicolumn{2}{c}{ Crecimiento postraumático } \\
\cline { 2 - 5 } & $\beta$ & $t$ & $\beta$ & $t$ \\
\hline (Constante) & 0.15 & $3.232^{* * *}$ & 0.05 & $12.065^{* * *}$ \\
Severidad subjetiva & 0.26 & $4.063^{* * *}$ & -0.1 & 1.21 \\
Rumiación negativa & -0.04 & -0.806 & 0.11 & -1.589 \\
Rumiación reflexiva & 0.11 & $2.016^{*}$ & 0.41 & $2.148^{*}$ \\
Rumiación deliberada & 0.38 & $5.922^{* * *}$ & -0.03 & $7.969^{* * *}$ \\
Rumiación intrusiva & & & & 0.582 \\
\hline
\end{tabular}

$* \mathrm{p}<0.05 ; * * * \mathrm{p}<0.001$

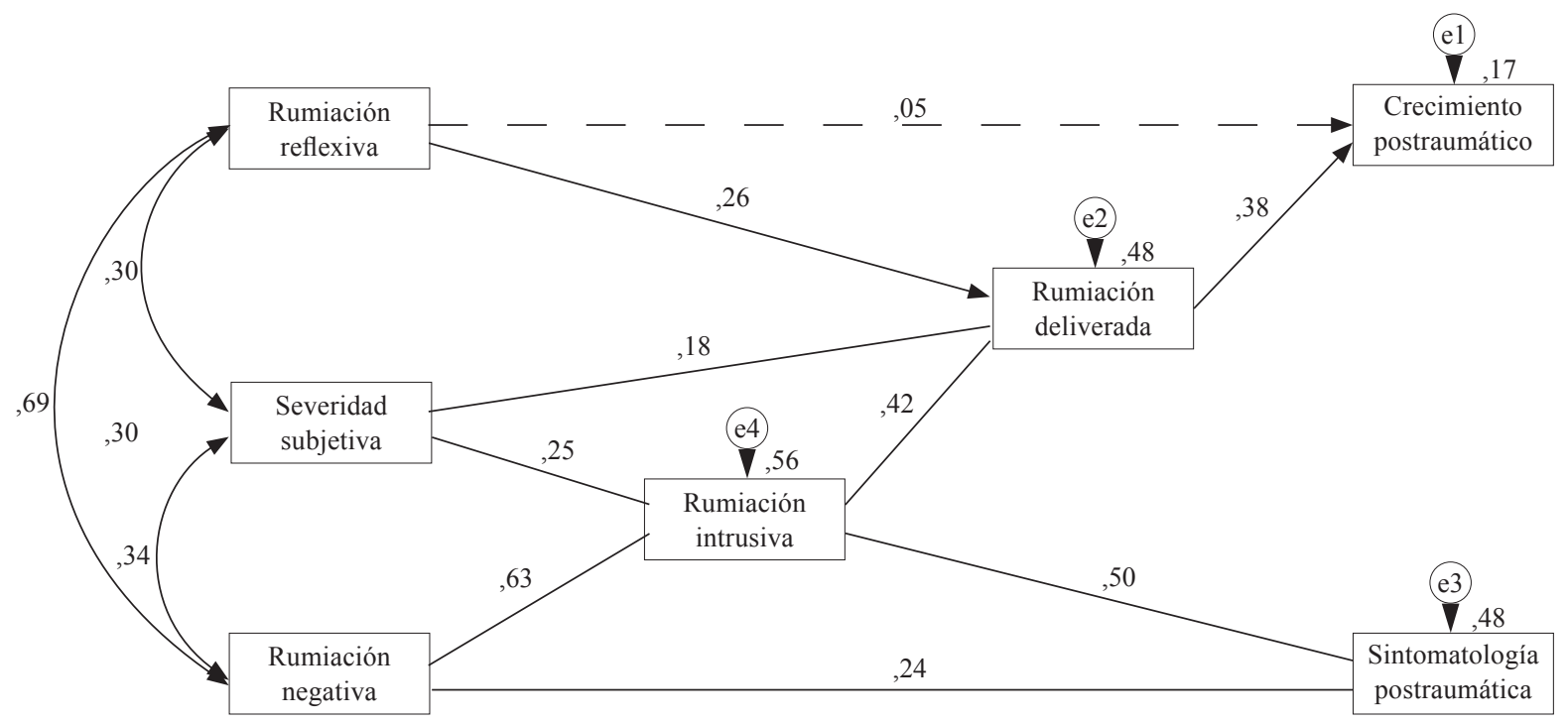

Figura 1. Modelo hipotetizado con coeficientes path estandarizados

que implica que los datos del estudio concuerdan con el modelo hipotetizado.

En la figura 1 se observan los coeficientes path entre las variables. Todos los efectos directos observados resultaron significativos, a excepción del efecto de la rumiación reflexiva sobre el CPT, que se representa en la figura con una línea intermitente. En este caso, se hipotetizó inicialmente una mediación parcial de la rumiación deliberada, sin embargo, los datos mostraron una mediación total.

Para evaluar las mediaciones, se realizó una estimación bootstrap de sesgo-corregido (2000 muestras bootstrap con intervalo de confianza de $95 \%$ ). En este caso, la mediación existe si el cero no está incluido en el intervalo de confianza (MacKinnon, Lockwood \& Williams, 2004), lo que indica que el efecto indirecto entre dos variables es significativo. Los análisis respaldaron la hipótesis de que la rumiacion deliberada media totalmente la relación de la severidad subjetiva, y las rumiaciones intrusiva y reflexiva con el CPT, y que la rumiación intrusiva media parcialmente la relación entre rumiación negativa y SPT, y totalmente la relación entre severidad subjetiva y SPT (tabla 4). 
Tabla 4

Los efectos indirectos estandarizados, el IC de $95 \%$ para los estimados (límite inferior y superior) y sus errores estándares

\begin{tabular}{lccc}
\hline \multicolumn{1}{c}{ Variables } & Efecto indirecto & IC (95\%) & EE \\
\hline Severidad subjetiva $\rightarrow$ Rumiación deliberada & 0.10 & $0.09 / 0.12$ & 0.005 \\
Severidad subjetiva $\rightarrow$ Crecimiento postraumático & 0.11 & $0.10 / 0.12$ & 0.006 \\
Severidad subjetiva $\rightarrow$ Sintomatología postraumática & 0.12 & $0.11 / 0.14$ & 0.006 \\
Rumiación reflexiva $\rightarrow$ Crecimiento postraumático & 0.10 & $0.09 / 0.11$ & 0.006 \\
Rumiación negativa $\rightarrow$ Rumiación deliberada & 0.26 & $0.24 / 0.28$ & 0.010 \\
Rumiación negativa $\rightarrow$ Crecimiento postraumático & 0.10 & $0.09 / 0.11$ & 0.005 \\
Rumiación negativa $\rightarrow$ Sintomatología postraumática & 0.31 & $0.30 / 0.33$ & 0.009 \\
Rumiación intrusiva $\rightarrow$ Crecimiento postraumático & 0.16 & $0.14 / 0.17$ & 0.008 \\
\hline
\end{tabular}

Tabla 5

Modelos de invarianza causal entre grupo de mujeres y hombres

\begin{tabular}{lccccc}
\hline & $\chi^{2}$ & gl & RMSEA & CFI & $\Delta$ CFI \\
\hline Modelo 1 & $284.831^{* * *}$ & 18 & 0.05 & 0.98 & - \\
Modelo 2 & $520.984 * * *$ & 27 & 0.05 & 0.98 & -0.01 \\
Modelo 3 & $710.959 * * *$ & 31 & 0.06 & 0.97 & -0.01 \\
Modelo 4 & $787.623 * * *$ & 35 & 0.06 & 0.96 & -0.01 \\
\hline
\end{tabular}

Modelo 1 = sin restricciones; modelo $2=\mathrm{M} 1+$ igualdad en pesos de regresión; modelo $3=\mathrm{M} 2+$ igualdad en los interceptos; modelo $4=\mathrm{M} 3+$ igualdad en las varianzas de error; $\mathrm{p}<0.001$.

Con base en estos resultados, que muestran diferencias de sexo en al menos tres variables del modelo, se decide realizar un análisis multigrupo con la finalidad de comparar si el modelo de sendero es equivalente en hombres y mujeres. Para ello, se realizó la evaluación secuencial de la invarianza causal a través de modelos anidados (Lévy \& Iglesias, 2007). El primer modelo no presentó restricciones; el segundo examinó la igualdad de los pesos de regresión; el tercero examinó la igualdad en los interceptos; el cuarto examinó la igualdad en las varianzas de error. El análisis mostró la existencia de invarianza en todos los niveles dado que el CFI disminuyó su valor en un nivel no superior al 0.01 con respecto al modelo anterior (Cheung \& Rensvold, 2002) y el RMSEA casi no sufrió variaciones, por lo que se puede concluir que el modelo de sendero presentado es equivalente en las dos submuestras (tabla 5).

\section{Discusión}

Al revisar las correlaciones entre las distintas variables incluidas en el estudio, podemos observar que son todas significativas y directas. Al tratarse de dos consecuencias supuestamente diferentes tras vivenciar una experiencia traumática, una negativa como la SPT y otra positiva como la CPT, resulta llamativo que ambas correlacionen directamente entre sí, con la severidad subjetiva y con todas las medidas de rumiación, sean consideradas adaptativas (reflexiva y deliberada) o maladaptativas (intrusiva y negativa). Sin embargo, esta situación resulta común en los estudios que han incluido estas 
variables (Alzugaray et al., 2015; García, Jaramillo et al., 2014; Morris \& Shakespeare-Finch, 2011; Stockton et al., 2011) y la explicación recurrente es que ambas aparecen como consecuencia de un evento traumático y del sufrimiento asociado, la SPT como una respuesta más patológica y la CPT como el aprendizaje que surge precisamente a partir de dicho sufrimiento (Calhoun y Tedeschi, 1998).

Al controlar a través de la RLM la influencia que la severidad y los distintos tipos de rumiación ejercen sobre la SPT y luego sobre el CPT, se observa que las principales influencias del SPT son las rumiaciones intrusiva y negativa - aunque de todos modos influyen la severidad subjetiva y rumiación deliberada-, lo cual se condice con las investigaciones realizadas sobre este tema (Allbaugh, 2013; Soo \& Sherman, 2015). A su vez, se observa que las variables que influyen en el CPT son las rumiaciones reflexiva y deliberada, lo que también coincide con la evidencia disponible (Stockton et al., 2011; Su \& Chen, 2015). Estos resultados se explicarían por la naturaleza adaptativa y maladaptativa de los distintos tipos de rumiación. En este caso, las rumiaciones negativa e intrusiva actúan como una estrategia de evitación cognitiva que inhibe la capacidad de un individuo para desplegar recursos de afrontamiento más adaptativos, lo cual dificulta la resolución de los problemas, y se asocia, por lo tanto, a mayores niveles de estrés y un estado de ánimo disfórico. Por otro lado, las rumiaciones reflexiva y deliberada constituyen un intento de entender el evento, encontrar posibles soluciones a los problemas derivados y descubrir posibles beneficios de haber estado expuesto a un evento altamente estresante (Walter \& Bates, 2012).

El modelo hipotético presentado, que permite evaluar a través de un análisis de sendero las distintas formas en las que la rumiación conduce a la SPT y al CPT, es quizás el aporte más significativo del presente estudio, pues permite concluir que existen vías separadas para uno u otro resultado, tal como planteaba Trapnell y Campbell (1999), actuando la rumiación intrusiva como mediador total de la severidad subjetiva y parcial de la rumiación negativa con la SPT. Así mismo, la rumiación deliberada actuaría como un mediador total de los otros procesos psicológicos evaluados, como la rumiaciones reflexiva y intrusiva, y la severidad subjetiva con el CPT. Estos efectos resultan coherentes con la teoría (Calhoun, Tedeschi, Cann \& Hanks, 2010) y con la evidencia científica previa (García, Jaramillo et al., 2014; García et al., 2015; Su \& Chen, 2015), y además abren interesantes posibilidades a la hora de abordar terapéuticamente el trauma para así reducir la posibilidad de SPT e incrementar la probabilidad de que la persona desarrolle un CPT.

Para Tedeschi y Blevins (2015), en el periodo inmediatamente posterior a un evento traumático, los pensamientos intrusivos entran inadvertidamente en la conciencia de un individuo, provocando la activación de la respuesta al estrés y las posibles experiencias de ansiedad, hipervigilancia, disociación y así sucesivamente. Sin embargo, ya sea en forma espontánea o a través de estrategias terapéuticas relacionadas a la exposición y el procesamiento cognitivo, en ocasiones la persona logra comprometerse en forma directa con sus pensamientos y sentimientos, favoreciendo la metacognición, es decir, la capacidad de observar y analizar los propios pensamientos. En otras palabras, es probable que la rumiación intrusiva inicial asociada al malestar emocional pueda mantenerse en el tiempo, prolongando la experiencia de estrés, o que pueda dar paso posteriormente a una rumiación más deliberada, lo que facilitaría el desarrollo del CPT (Calhoun et al., 2010). Esto es lo que se observa en el análisis de sendero del presente estudio.

El paso de una rumiación intrusiva a una más deliberada proporciona un espacio para la toma de perspectiva y de los procesos de reevaluación potenciales, permitiendo en forma gradual articular una nueva narrativa, en la cual el trauma adquiere un sentido y se encamina hacia su resolución. De ese modo, toda la experiencia puede ser vista como 
significativa, ya que se desarrolla un aprendizaje en relación con la experiencia, permitiendo que el sobreviviente de trauma pueda proceder en la vida con un sentido de bienestar eudaimónico. De ese modo, la promoción de una rumiación deliberada, obtenida espontáneamente por los participantes del presente estudio, podría ser una de las más importantes vías para conducir al CPT. La razón de ello es que dicha rumiación es semejante al procesamiento cognitivo que se incentiva en terapias cognitivas de segunda y tercera generación, como la restructuración cognitiva y el mindfulness (Garland, Farb, Goldin \& Fredrickson, 2015), o en las terapias sistémicas a través de la prescripción del síntoma, incentivando a las personas a que los pensamientos disruptivos que los afectan constantemente puedan manifestarse de manera voluntaria y controlada (García et al., 2014). Si bien se ha señalado que la reestructuración cognitiva y el mindfulness promueven el cambio mediante mecanismos diferentes, ambos parecen incrementar las habilidades metacognitivas de la persona, característica que compartirían con la rumiación deliberada (Tedeschi \& Blevins, 2015).

En cuanto a las limitaciones del presente estudio, podemos señalar que este es de carácter transversal, por lo que las relaciones causales presentadas son hipotéticas, lo que abre la necesidad de realizar estudios longitudinales que permitan confirmar las hipótesis planteadas. Por otro lado, la heterogeneidad de eventos a los que estas personas estuvieron expuestas, si bien es una fortaleza toda vez que representan mejor la diversidad de eventos estresantes de la población general, hace suponer que estos resultados, en muestras más específicas, podrían ser distintos, por lo que se recomienda continuar investigando esas relaciones en personas expuestas a eventos estresantes más delimitados.

Este estudio, al reconocer las probables vías que siguen los distintos procesos de rumiación y que potencialmente conducen a SPT o CPT, además de observar el rol mediador de las rumiaciones deliberada e intrusiva para estos dos tipos de con- secuencia, permitirá desarrollar estrategias que ayuden a la elaboración cognitiva de las intrusiones luego del primer momento tras el trauma o que fomenten formas de pensamiento más deliberadas, estrategias que requieren ser evaluadas a través de diseños experimentales. Así mismo, este estudio contribuye a la comprensión de la consecuencia que genera la transición desde formas intrusivas de pensamiento hacia formas más controladas y constructivas, aun cuando este estudio no entrega una respuesta respecto a qué variables contribuyen a que se produzca esa transición, pudiendo ser la oportunidad de conectarse con redes sociales de apoyo, como señalan Tedeschi y Calhoun (2004), $\mathrm{u}$ otros procesos que requieren ser incorporados a modelos más complejos, como la autorregulación emocional (Curci, Soleti, Lanciano, Doria \& Rimé, 2015), la autorrevelación (Dong, Gong, Jiang, Deng \& Liu, 2015) o los niveles de religiosidad-espiritualidad (Bray, 2013), entre otros.

\section{Agradecimientos}

Este trabajo fue apoyado por CONICYT/FONDECYT n. ${ }^{\circ} 1180134$ para Felipe E. García. Los autores agradecen a la Mutual de Seguridad de la Cámara Chilena de la Construcción, en especial al doctor Marcelo Olivares, doctor Carlos Brain, doctor Rodrigo Campos, Marly Céspedes, Miriam Ramos y María Carolina Espinoza, sin cuyo apoyo no podría haberse desarrollado este proyecto.

\section{Referencias}

Allbaugh, L. J. (2013). Rumination in the context of the centrality of stressful events (tesis inédita de doctorado). Miami University, Miami, Estados Unidos.

Alzugaray, C., García, F., Reyes, A. \& Álvarez, R. (2015). Propiedades psicométricas de una versión breve de la escala de rumiación relacionada a un evento en población chilena afectada por 
eventos altamente estresantes. Ajayu, 13(2), 183-198.

American Psychiatric Association (2000). Manual diagnóstico y estadístico de los trastornos mentales, texto revisados: DSMIV-TR. Washington DC: Masson.

Arbuckle, J. L. (2003). Amos user's guide. Chicago, IL: SmallWaters.

Aron, A. \& Aron, E. (2001). Estadística para psicología. Buenos Aires: Pearson Education.

Avilés, P., Cova, F., Bustos, C. \& García, F. (2014). Afrontamiento y rumiación frente a eventos adversos y crecimiento postraumático en estudiantes universitarios. Liberabit, 20(2), 281-292.

Boals, A. \& Schuettler, D. (2009). PTSD symptoms in response to traumatic and non-traumatic events: The role of respondent perception and A2 criterion. Journal of Anxiety Disorders, 23, 458-462. DOI: 10.1016/j.janxdis.2008.09.003

Bray, P. (2013). Bereavement and transformation: a psycho-spiritual and post-traumatic growth perspective. Journal of Religion and Health, 52(3), 890-903. DOI: 10.1007/s10943-0119539-8

Calhoun, L. G., Cann, A. \& Tedeschi, R. G. (2010). The posttraumatic growth model: socio-cultural considerations. En T. Weiss \& R. Berger (eds.), Posttraumatic growth and culturally competent practice: lessons learned from around the globe (pp. 1-14). Hoboken, NJ: Wiley.

Calhoun, L. G. \& Tedeschi, R. G. (1998). Beyond recovery from trauma: implications for clinical practice and research. Journal of Social Issues, 54, 357-371. DOI: 10.1111/00224537.701998070

Calhoun, L. G., Tedeschi, R. G., Cann A. \& Hanks, E. (2010). Positive outcomes following bereavement: paths to posttraumatic growth. Psychologica Belgica, 50, 125-143. DOI: 10.5334/ pb-50-1-2-125

Cann, A., Calhoun, L. G., Tedeschi, R. G., Taku, K., Vishnevsky, T., Triplett, K. \& Danhauer, S. (2010). A short form of the Posttraumatic
Growth Inventory. Anxiety, Stress, \& Coping, 23, 127-137. DOI: 10.1080/10615800903094273

Cann, A., Calhoun, L., Tedeschi, R., Triplett, K., Vishnevsky, T. \& Lindstrom, C. (2011). Assessing posttraumatic cognitive processes: the event related rumination inventory. Anxiety, Stress, \& Coping, 24(2), 137-156. DOI: 10.1080/10615806.2010.529901

Cheung, G. W. \& Rensvold, R. B. (2002). Evaluating goodness-of-fit indexes for testing measurement invariance. Structural Equation Modeling, 9(2), 233-255. DOI: 10.1207/S15328007SEM0902_5

Connor, K. \& Davidson, J. (2001). SPRINT: a brief global assessment of post-traumatic stress disorder. International Clinical Psychopharmacology, 16(5), 279-284. DOI: 10.1097/00004850200109000-00005

Cova, F., Rincón, P. \& Melipillán, R. (2007). Rumiación y presencia de sintomatología ansiosa y depresiva en adolescentes. Revista Mexicana de Psicología, 24(2), 175-183.

Cova, F., Rincón, P. \& Melipillán, R. (2009). Reflexión, rumiación negativa y desarrollo de sintomatología depresiva en adolescentes de sexo femenino. Terapia Psicológica, 27(2), 155160. DOI: $10.4067 / \mathrm{S} 0718-48082009000200001$

Curci, A., Soleti, E., Lanciano, T., Doria, V. \& Rimé, B. (2015). Balancing emotional processing with ongoing cognitive activity: the effects of task modality on intrusions and rumination. Frontiers in Psychology, 6. DOI: 10.3389/ fpsyg.2015.01275

Danhauer, S. C., Russell, G. B., Tedeschi, R. G., Jesse, M. T., Vishnevsky, T., Daley, K., Carroll, S., Triplett, K. N., Calhoun, L. G., Cann, A. \& Powell, B. L. (2013). A longitudinal investigation of posttraumatic growth in adult patients undergoing treatment for acute leukemia. Journal of Clinical Psychology in Medical Settings, 20(1), 13-24. DOI: 10.1007/s10880-012-9304-5

Dong, C., Gong, S., Jiang, L., Deng, G. \& Liu, X. (2015). Posttraumatic growth within the first three months after accidental injury in China: 
the role of self-disclosure, cognitive processing, and psychosocial resources. Psychology, Health \& Medicine, 20(2), 154-164. DOI: $10.1080 / 13548506.2014 .913795$

García, F., Cova, F., Rincón, P. \& Vázquez, C. (2015). Trauma or growth after a natural disaster? The mediating role of rumination processes. European Journal of Psychotraumathology, 6, 1-10. DOI: 10.3402/ejpt.v6.26557

García, F., Cova, F., Rincón, P., Vázquez, C. \& Páez, D. (2016). Coping, rumination and posttraumatic growth in people affected by an earthquake. Psicothema, 28(1), 59-65. DOI: 10.7334/psicothema2015.100

García, F., Duque, A. \& Cova, F. (2017). The four faces of rumination to stressful events: a psychometric analysis. Psychological Trauma: Theory, Research, Practice, and Police, 9(6), 758-765. DOI: 10.1037/tra0000289

García, F., Jaramillo, C., Martínez, A. M., Valenzuela, I. \& Cova, F. (2014). Respuestas psicológicas ante un desastre natural: Estrés y crecimiento postraumático. Liberabit, 20, 121-130.

García, F., Reyes, A. \& Cova, F. (2014). Severidad del trauma, optimismo, crecimiento postraumático y bienestar en sobrevivientes de un desastre natural. Universitas Psychologica, 13(2), 1524. DOI: $10.11144 / J a v e r i a n a . U P S Y 13-2 . s t o p$

García, F. \& Wlodarczyk, A. (2016). Psychometric properties of the Post-traumatic Growth Inventory - Short Form among Chilean adults. Journal of Loss and Trauma, 21(4), 303-314. DOI: 10.1080/15325024.2015.1108788

Garland, E. L., Farb, N. A., R. Goldin, P. \& Fredrickson, B. L. (2015). Mindfulness broadens awareness and builds eudaimonic meaning: a process model of mindful positive emotion regulation. Psychological Inquiry, 26(4), 293314. DOI: 10.1080/1047840X.2015.1064294

Hafstad, G., Gil Rivas, V., Kilmer, R. \& Raeder, S. (2010). Posttraumatic growth among norwegian children and adolescents following a natural disaster. American Journal of Orthopsychiatry, 80, 248-257. DOI: 10.1037/a0023236

Hu, L. \& Bentler, P. (1999). Cutoff criteria for fit indexes in covariance structure analysis: conventional criteria versus new alternatives. Structural Equation Modeling, 6, 1-55. DOI: 10.1080/10705519909540118

Kane, J. (2009). An examination of a two-Factor model of rumination and its impact on the relationship between posttraumatic growth and Posttraumatic Stress Disorder (PTSD) (tesis inédita de doctorado). George Mason University, Fairfax, Estados Unidos.

Kilmer, R. P. \& Gil- Rivas, V. (2010). Exploring posttraumatic growth in children impacted by $\mathrm{Hu}-$ rricane Katrina: correlates of the phenomenon and developmental considerations. Child Development, 81, 1211-1227. DOI: 10.1111/j.14678624.2010.01463.x

Leiva-Bianchi, M. C. \& Gallardo, I. (2013). Validation of the Short Posttraumatic Stress Disorder Rating Interview (SPRINT-E) in a sample of people affected by F-27 Chilean earthquake and tsunami. Anales de Psicología, 29(2), 328-334. DOI: 10.6018/analesps.29.2.130681

Lévy, P. \& Iglesias, V. A. (2007). Invarianza causal con muestras múltiples. En J. P. Lévy \& J. Varela (eds.), Modelización con estructuras de covarianzas en ciencias sociales (pp. 279-318). La Coruña: Netbiblo.

Lindstrom, C. M., Cann, A., Calhoun, L. G. \& Tedeschi, R. G. (2013). The relationship of core belief challenge, rumination, disclosure, and sociocultural elements to posttraumatic growth. Psychological Trauma: Theory, Research, Practice, and Policy, 5(1), 50-44. DOI: 10.1037/ a0022030

MacKinnon, D. P., Lockwood, C. M. \& Williams, J. (2004). Confidence limits for the indirect effect: Distribution of the product and resampling methods. Multivariate Behavioral Research, 39, 99-128. DOI: 10.1207/s15327906mbr3901_4 
McCaslin, S. E., Zoysa, P., Butler, L. D., Hart, S., Marmar, C. R., Metzler, T. J. \& Koopman, C. (2009). The relationship of posttraumatic growth to peritraumatic reactions and posttraumatic stress symptoms among Sri Lankan University students. Journal of Traumatic Stress, 22, 334339. DOI: $10.1002 /$ jts. 20426

Morris, B. A. \& Shakespeare-Finch, J. (2011). Rumination, post-traumatic growth, and distress: structural equation modelling with cancer survivors. Psycho-Oncology, 20, 1176-1183. DOI: 10.1002/pon. 1827

Morris, B., Shakespeare-Finch, J., Rieck, M. \& Newberry, J. (2005). Multidimensional nature of posttraumatic growth in an Australian population. Journal of Traumatic Stress, 18, 575-585. DOI: $10.1002 /$ jts. 20067

Nolen-Hoeksema, S. (1987). Sex differences in unipolar depression: evidence and theory. Psychological Bulletin, 101(2), 259-282. DOI: 10.1037/0033-2909.101.2.259

Norris, F., Hamblen, J., Brown, L. \& Schinka, J. (2008). Validation of the Short Posttraumatic Stress Disorder Rating Interview (expanded version, Sprint-E) as a measure of postdisaster distress and treatment need. American Journal of Disaster Medicine, 3, 201-212.

Papageorgiou, C. \& Wells, A. (2004). Depressive rumination: nature, theory and treatment. Nueva Jersey: John Wiley \& Sons.

Pérez, E., Medrano, L. A. \& Sánchez Rosas, J. (2013). El Path Analysis: conceptos básicos y ejemplos de aplicación. Revista Argentina de Ciencias del Comportamiento, 5(1), 52-66.

Soo, H. \& Sherman, K. A. (2015). Rumination, psychological distress and post-traumatic growth in women diagnosed with breast cancer. PsychoOncology, 24(1), 70-79. DOI: 10.1002/pon.3596

Stockton, H., Hunt, N. \& Joseph, S. (2011). Cognitive processing, rumination, and posttraumatic growth. Journal of Traumatic Stress, 24(1), 85-92. DOI: $10.1002 /$ jts.20606
Su, Y. J. \& Chen, S. H. (2015). Emerging posttraumatic growth: a prospective study with pre-and posttrauma psychological predictors. Psychological Trauma: Theory, Research, Practice, and Policy, 7(2), 103. DOI: 10.1037/tra0000008 Sutin, A., Costa, P., Wethington, E. \& Eaton, W. W. (2010). Perceptions of stressful life events as turning points are associated with self-rated health and psychological distress. Anxiety, Stress and Coping, 23(5), 479-492. DOI: $10.1080 / 10615800903552015$

Taku, K., Cann, A., Tedeschi, R. G. \& Calhoun, L. G. (2009). Intrusive versus deliberate rumination in posttraumatic growth across US and Japanese samples. Anxiety, Stress \& Coping, 2(22), 2936. DOI: $10.1080 / 10615800802317841$

Tedeschi, R. G. \& Calhoun, L. G. (1996). The Posttraumatic Growth Inventory: measuring the positive legacy of trauma. Journal of Traumatic Stress, 9, 455-471. DOI: 10.1002/jts.2490090305

Tedeschi, R. G. \& Calhoun, L. G. (2004). Posttraumatic growth: conceptual foundations and empirical evidence. Psychological Inquiry, 15(1), 1-18. DOI: 10.1207/s15327965pli1501_01

Tedeschi, R. G. \& Blevins, C. L. (2015). From mindfulness to meaning: implications for the theory of posttraumatic growth. Psychological Inquiry, 26(4), 373-376. DOI: 10.1080/1047840X.2015.1075354

Trapnell, P. D. \& Campbell, J. D. (1999). Private self-consciousness and the five-factor model of personality: distinguishing rumination from reflection. Journal of Personality and Social Psychology, 76(2), 284-304. Dor: 10.1037/00223514.76.2.284

Treynor, W., González, R. \& Nolen-Hoeksema, S. (2003). Rumination reconsidered: a psychometric analysis. Cognitive Therapy and Research, 27(3), 247-259. DOI: 10.1023/A:1023910315561

Triplett, K. N., Tedeschi, R. G., Cann, A., Calhoun, L. G. \& Reeve, C. L. (2012). Posttraumatic growth, meaning in life, and life satisfaction in response to trauma. Psychological Trauma: 
Theory, Research, Practice, and Policy, 4(4), 400-410. DOI: 10.1037/a0024204

Vázquez, C., Castilla, C., y Hervás, G. (2009). Reacciones ante el trauma: resistencia y crecimiento. En E. Fernández-Abascal (ed.), Las emociones positivas (pp. 375-392). Madrid: Pirámide.

Walter, M. \& Bates, G. (2012). Posttraumatic growth and recovery from post-traumatic stress disor- der. En V. Olisah (ed.), Essential notes in psychiatry (pp. 143-186). Rijeka: InTech.

Yu, C. Y. (2002). Evaluating cutoff criteria of model fit indices for latent variable models with binary and continuous outcomes (tesis inédita de doctorado). University of California, Los Angeles, Estados Unidos. Recuperado de http://www. statmodel.com/download/Yudissertation.pdf

Recibido: julio 4, 2016

Aprobado: febrero 23, 2018 
